\title{
Estrategias metodológicas para la enseñanza y el aprendizaje de la geometría, utilizadas por docentes de segundo ciclo, con la finalidad de generar una propuesta metodológica atingente a los contenidos
}

\author{
Methodological strategies for the teaching and learning of geometry, used by teachers in \\ second cycle, in order to generate a methodological approach adequate to contents
Estratégias metodológicas para o ensino e a aprendizagem da geometria, utilizadas por docentes de segundo ciclo, com a finalidade de gerar uma proposta metodológica que atinja os conteúdos

\section{Roxana Fabres Fernández.}

Universidad de La Frontera, Temuco, 64948262, rfabres2014@gmail.com

\section{RESUMEN}

Esta investigación de carácter cualitativa responderá a interrogantes ¿Cuáles son las estrategias metodológicas para la enseñanza y el aprendizaje de la geometría? Y ¿Qué propuesta metodológica se puede generar para enseñar los contenidos de geometría? Los hallazgos obtenidos fueron que los docentes realizan actividades de definición de conceptos y dibujos para trabajar los contenidos conceptuales, los procedimientos son desarrollados en base a una enseñanza tradicional; clases expositivas, explicación de ejercicios para posterior replicación por los estudiantes, en éstas priman las guías y textos de estudios, evidenciando una ausencia de variedad de materiales concretos y actividades lúdicas. Algunos docentes declaran no conocer el modelo de Van Hiele, por lo cual no es utilizado en sus prácticas, y los que señalan conocerlo, no lo usan en su totalidad. Al evidenciar ausencias metodologías, se proponen actividades basadas en el modelo de Van Hiele, así como la incorporación de material concreto y actividades lúdicas.

Palabras clave: geometría, modelo de Van Hiele, material concreto, habilidades, estrategias de enseñanza.

\section{ABSTRACT}

This qualitative research will answer the questions: What are the methodological strategies for the teaching and learning of geometry? and, What methodology can be created to teach the contents of geometry? The findings obtained show that teachers carry out activities for defining concepts and drawings to work the conceptual content, procedures are developed based on traditional teaching, exhibition classes, and explanation of exercises for further replication by students. All of them based on guides and texts of studies, demonstrating a lack of variety of concrete materials and fun activities. Some teachers declare not knowing Van Hiele model and so they do not used it in their practice, and those who point to know him, do not apply it entirely. After evidencing methodological absences, activities based on Van Hiele model are proposed, as well as the incorporation of concrete material and fun activities.

Key words: geometry, Van Hiele model, concrete material, skills, teaching strategies.

\section{RESUMO}

Esta pesquisa qualitativa responderá às perguntas: Quais são as estratégias metodológicas para o ensino e a aprendizagem de geometria? e Qual proposta metodológica pode ser criada para ensinar os conteúdos de geometria? As descobertas obtidas foram que professores realizam atividades de definição de conceitos e desenhos para trabalhar conteúdos conceituais, os procedimentos são desenvolvidos com base em um ensino tradicional: aulas 
Estudios Pedagógicos XLII, Nº 1: 87-105, 2016

ESTRATEGIAS METODOLÓGICAS PARA LA ENSEÑANZA Y EL APRENDIZAJE DE LA GEOMETRÍA, UTILIZADAS POR DOCENTES DE SEGUNDO CICLO, CON LA FINALIDAD DE GENERAR UNA PROPUESTA METODOLÓGICA ATINGENTE A LOS CONTENIDOS

expositivas, explicação de exercícios para a posterior explicação pelos estudantes, nestes destacam-se os livros e textos de estudos, demonstrando uma ausência de variedade de materiais concretos e atividades lúdicas. Alguns professores declaram não conhecer o modelo de Van Hiele, o que justifica o não emprego dele em suas práticas e, aqueles que afirmam conhecê-lo, não o empregam em sua totalidade. Ao evidenciar ausências metodológicas, propõem-se atividades baseadas no modelo de Van Hiele, bem como a incorporação do material concreto e de atividades lúdicas nas aulas.

Palavras-chave: geometria, modelo de Van Hiele, material concreto, habilidades, estratégias de ensino.

\section{INTRODUCCIÓN}

La geometría, junto a la teoría de números, es una de las ramas más antiguas de la matemática. Si por un momento se restringe el término para referirse a lo que los antiguos griegos entendían como tal, se puede decir que su objeto de estudio está íntimamente arraigado en la forma de concebir la realidad. Toda la información que se recibe del mundo que nos rodea, todo lo que se ve, oye y toca, es procesado en primera instancia en términos geométricos.

A través de la historia, la geometría ha sido enfocada de diferentes maneras. Para los egipcios, fue práctica y utilitaria: medían los terrenos irregulares aplicando la triangulación. Para los griegos, en cambio, estuvo relacionada con el enriquecimiento del espíritu (Riveros y Zanocco, 1988). En nuestra época, la geometría se asocia a la astronomía, a la arquitectura y a muchas otras ciencias, artes y técnicas.

Para lograr que nuestros estudiantes se interesen en la geometría, hay que tener presente que el medio que los rodea está lleno de elementos geométricos. Sólo necesitan un poco de observación dirigida para apreciarlos; el aprendizaje de la geometría se hace más fácil y entretenido, si los alumnos pueden trabajar con materiales concretos, tener la experiencia de tocar y palpar; es necesario estructurar una secuencia programática de acuerdo al desarrollo intelectual de los alumnos.

La geometría puede llegar a estimular el interés por el aprendizaje de esta ciencia, ya que muestra de una manera distinta la realidad que rodea al alumno, da oportunidad de desarrollar habilidades imaginativas y creativas a través del trabajo con las formas. Una meta deseable es que el alumno llegue a recrearse con el aprendizaje de la geometría. Durante toda su vida, el alumno interactuará con objetos concretos en un espacio físico; tanto lo real como las interacciones pueden ser matematizadas, es decir, representadas esquemáticamente como entes geométricos.

El espacio físico no es la única fuente de matematización, pero su importancia debe ser resaltada, dado que los alumnos elaboran el espacio lógico-matemático a partir de las acciones que efectúan sobre objetos concretos en el espacio real.

Numerosos trabajos (cit. en Bressan et al., 2010) destacan la postergación que sufre esta rama de la matemática en las escuelas, en favor de la enseñanza de otros tópicos de la aritmética en primaria o de la aritmética y del álgebra en secundaria, los cuales ocupan el mayor tiempo de la enseñanza matemática escolar.

La presente investigación se enfocó en conocer estrategias metodológicas para la enseñanza y el aprendizaje de la geometría, utilizadas por docentes de segundo ciclo, con la finalidad de generar una propuesta metodológica en base a actividades lúdicas, e incorporando material concreto, que sean atingentes a los contenidos programáticos de ese ciclo educativo en Chile. 


\section{MARCO TEÓRICO}

La matemática, como una expresión de la mente humana, refleja la voluntad activa, la razón contemplativa y el deseo de perfección estética. Sus elementos básicos son: lógica e intuición, análisis y construcción, generalidad y particularidad. Aunque diversas concepciones han destacado aspectos diferentes, es únicamente el juego de estas fuerzas opuestas y la lucha por su síntesis lo que constituye la vida, la utilidad y el supremo valor de la ciencia matemática.

Gran parte de la matemática ha tenido su origen en necesidades más o menos prácticas, pero siempre a través de su desarrollo ésta transciende los confines de la utilidad inmediata. (Duval, 2001). Las representaciones matemáticas, es decir, los contenidos que habitualmente se enseñan en el aula, son considerados construcciones sociales, dado que el conocimiento matemático representa las experiencias materiales de personas que interactúan en situaciones particulares, culturas y períodos históricos determinados:

[...] la ciencia forma parte de la cultura [...] Significa simple y sencillamente que la ciencia es una construcción humana, una institución progresivamente elaborada, históricamente condicionada e inseparable de las demás instituciones o actividades humanas... (Cit. en Villella, 2001:15)

\subsection{LA GEOMETRÍA EN LA MATEMÁTICA DE LA ESCUELA}

Villella (2001) plantea que enseñar geometría en la escuela supone ofrecer a los alumnos la posibilidad de: describir, entender e interpretar el mundo real y sus fenómenos; resolver una amplia variedad de problemas que implican usos de estimación, aproximación y medición; acceder paulatinamente, mediante el uso de técnicas y de las explicaciones sobre ellas se pueden dar, a un modo de teoría axiomática; formular conjeturas y preguntas; proponer pruebas, estrategias; elaborar refutaciones, ejemplos y contraejemplos de los fenómenos que se describen y modelizan primero, para aplicar las conclusiones a otros fenómenos; recuperar la capacidad de asombro y de análisis de lo visual, de la imagen; construir esquemas básicos de respuestas a situaciones cotidianas provenientes tanto del medio social como de las otras áreas de estudio, así como de la propia matemática, que involucran la conceptualización de lo espacial.

Para lograr que los estudiantes se interesen en la geometría, hay que tener presente que: el medio que los rodea está lleno de elementos geométricos; sólo necesitan un poco de observación dirigida para apreciarlos; la geometría aplicada es tan importante como la geometría pura; el arte, la música, la arquitectura, apoyará esta idea; el aprendizaje de la geometría se hace más fácil, y entretenido, si los estudiantes pueden trabajar con materiales concretos, para tener la experiencia de tocar y palpar; que es necesario estructurar una secuencia programática, de acuerdo al desarrollo intelectual de los estudiantes (Villella, 2001).

El docente que enseña geometría debe tener presente que el fin de su enseñanza es desarrollar en los estudiantes ciertas habilidades que les permitan: analizar características y propiedad de las figuras geométricas en tres, dos y una dimensión, y desarrollar argumentos para relacionarlas; usar sistemas de representación para lograr la localización espacial; aplicar transformaciones para analizar situaciones matemáticas; usar la visualización y el 
Estudios Pedagógicos XLII, Nº 1: 87-105, 2016

ESTRATEGIAS METODOLÓGICAS PARA LA ENSEÑANZA Y EL APRENDIZAJE DE LA GEOMETRÍA, UTILIZADAS POR DOCENTES DE SEGUNDO CICLO, CON LA FINALIDAD DE GENERAR UNA PROPUESTA METODOLÓGICA ATINGENTE A LOS CONTENIDOS

razonamiento espacial para la construcción de modelos geométricos con los cuales explicar fenómenos reales y situaciones matemáticas particulares.

Entre esas habilidades (cit. en Villella, 2001:80), se pueden mencionar aquellas relacionadas con: lo visual, la construcción, la comunicación, el desarrollo del pensamiento y la transferencia, que comprende habilidades para interpretar y analizar el mundo físico, y actuar en su entorno, desarrollando modelos que pueden interpretarse con los contenidos estudiados en geometría.

\subsection{EL MODELO DE RAZONAMIENTO GEOMÉTRICO DE VAN HIELE}

Van Hiele (1957), plantea niveles de razonamiento geométrico, lo que significa que quien aprende puede situarse respecto de la geometría en un nivel de razonamiento distinto del que presenta para el álgebra o el cálculo.

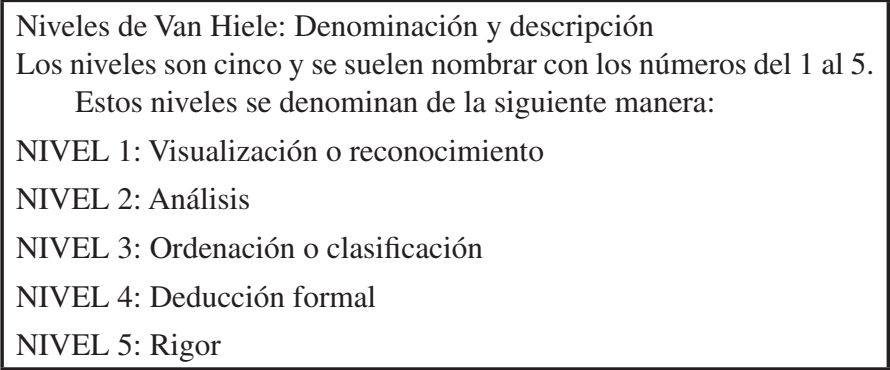

A continuación se presenta cómo organizar las actividades dentro de una unidad didáctica, es decir, qué tipo de actividades hacer conforme al desarrollo de la unidad. En su trabajo, Van Hiele enfatiza la idea que "el paso de un nivel a otro depende más de la enseñanza recibida que de la edad o madurez" (Villella, 2001: 210), es decir, da una gran importancia a la organización del proceso de enseñanza-aprendizaje, así como a las actividades diseñadas y los materiales utilizados.

Las fases que postula en su modelo son cinco y que, a continuación, se describen:

Fase 1a: Preguntas/Información

Fase 2a: Orientación Dirigida

Fase 3a: Explicación (Explicitación)

Fase 4a: Orientación Libre

Fase 5a: Integración

\section{SUPUESTOS}

Los docentes de matemática de segundo ciclo, utilizan estrategias orientadas a la enseñanza dirigida de contenidos conceptuales y demostración de aquéllos, los cuales son reproducidos por los estudiantes. 
Las estrategias empleadas por los docentes para la enseñanza de la geometría no potencian habilidades de transferencia, impidiendo con ello la utilización de las matemáticas en los contextos reales de los estudiantes.

Una propuesta metodológica efectiva es aquella que esté orientada a tareas de conceptualización, investigación y demostración, permitiendo trabajar las habilidades de visualización, dibujo, comunicación, razonamiento lógico y transferencia, basadas en los niveles de razonamiento propuestos por Van Hiele y un enfoque de resolución de problemas.

\section{OBJETIVO GENERAL}

Analizar las estrategias metodológicas para la enseñanza y el aprendizaje de la geometría, utilizadas por docentes de segundo ciclo de la comuna de Temuco y Padre Las Casas, con la finalidad de generar propuestas metodológicas atingente a los contenidos del área, a través de la incorporación de actividades lúdicas y material concreto.

\section{OBJETIVOS ESPECIFICOS}

Identificar las metodologías de enseñanza y aprendizaje de la geometría, que utilizan docentes de segundo ciclo de la comuna de Temuco y Padre Las Casas.

Describir las metodologías utilizadas por docentes para la enseñanza y el aprendizaje de la geometría, con la finalidad de determinar aquellos contenidos para los cuales se requiere proponer nuevas estrategias metodológicas.

Proponer nuevas estrategias metodológicas para la enseñanza y el aprendizaje de la geometría, basadas en el modelo de Van Hiele, incorporando actividades lúdicas y material concreto.

\section{METODOLOGÍA}

\subsection{PARADIGMA}

La presente investigación se orientó en un paradigma interpretativo/cualitativo, el cual, según Stratuss y Corbin (cit. en Sandín, 2003:121), se entiende como cualquier tipo de investigación que produce resultados a los que no se han llegado por procedimientos estadísticos u otro tipo de cuantificación. Puede referirse a investigaciones acerca de la vida de las personas, historias, comportamientos y también al funcionamiento organizativo, movimientos sociales, relaciones e interacciones. Así, se podrá conocer la realidad existente respecto a las estrategias metodológicas que utilizan docentes de segundo ciclo, en la enseñanza de la geometría, y a partir de ésto generar una propuesta metodológica.

\subsection{APROXIMACIÓN METODOLÓGICA}

El diseño de la investigación se basó en un estudio de caso en tanto que tipo de investigación educativa, que "constituye un método de investigación para el análisis de la realidad 
Estudios Pedagógicos XLII, Nº 1: 87-105, 2016

ESTRATEGIAS METODOLÓGICAS PARA LA ENSEÑANZA Y EL APRENDIZAJE DE LA GEOMETRÍA, UTILIZADAS POR DOCENTES DE SEGUNDO CICLO, CON LA FINALIDAD DE GENERAR UNA PROPUESTA METODOLÓGICA ATINGENTE A LOS CONTENIDOS

social de gran importancia en el desarrollo de las ciencias sociales y humanas, siendo la forma más pertinente y natural de investigación orientada desde una perspectiva cualitativa" (cit. en Sadín 2003: 174). Esta aproximación metodológica es considerada como la más adecuada para el presente estudio, dado que la línea investigativa apunta hacia la observación y el análisis del contexto educativo, sin alterar la realidad observada dentro del aula. Esta investigación implica un proceso de indagación que se caracteriza por un examen detallado, comprensivo, sistemático y en profundidad del caso objeto de estudio. (cit. en Sandín, 2003).

En relación al propósito del estudio y las características de la investigación, ésta se encontró dentro de la modalidad de estudio colectivo de caso, en donde "el interés se centra en la indagación de un fenómeno, población o condición general. El estudio no se focalizó en un caso concreto, sino en un determinado conjunto de casos" (cit. en Sandín, 2003:176).

En consideración a lo anterior, este enfoque fue el más adecuado para la investigación, ya que las realidades con las cuales se trabajó, arrojó teorías levantadas en base a la práctica y las interacciones que los sujetos mantenían entre sí, dependiendo de los diferentes contextos. Asimismo, al tener datos descriptivos, fue posible realizar un posterior análisis interpretativo de la realidad observada, de manera más profunda, a través de categorizaciones que permitió una comprensión completa de los fenómenos.

\subsection{PARTICIPANTES}

La población estará compuesta por profesores de segundo ciclo de la asignatura de matemática de establecimientos educacionales de la comuna de Temuco y Padre Las Casas. De dicha población, las unidades de análisis están compuestas principalmente por 4 docentes que ejercen docencia en dicho contexto educativo. Los criterios de inclusión para los profesores que participaron de la investigación son: estar ejerciendo la asignatura de matemática en segundo ciclo básico, en el establecimiento educativo, facilidad de acceso al campo de estudio, disponibilidad de los profesores y de la institución educativa para colaborar con la investigación.

\subsection{TÉCNICAS E INSTRUMENTOS DE RECOLECCIÓN DE DATOS}

Con el fin de lograr los objetivos planteados en la investigación, en cuanto a identificar las metodologías de enseñanza y aprendizaje de la geometría utilizadas por docentes de cursos de segundo ciclo, se utilizó como instrumento la entrevista semiestructurada.

Según Latorre, la entrevista "posibilita obtener información sobre acontecimientos y aspectos subjetivos de la persona, creencias y actitudes, opiniones, valores o conocimientos que de otra manera no estarían al alcance del investigador" (2003: 70).

Se analizaron las planificaciones de los profesores para contrastar la información obtenida de las entrevistas semiestructuradas. No fue posible realizar observación de clase, porque las fechas de programación de los contenidos de geometría no correspondían a los períodos de la investigación.

\section{ANÁLISIS E INTERPRETACIÓN DE LOS RESULTADOS}

Para este análisis, se ha seguido una metodología de naturaleza cualitativa, cuyo procedimiento inductivo está destinado a conocer las estrategias de enseñanza-aprendizaje 
que aplican los maestros para enseñar los contenidos que se tratan en geometría. Los resultados se obtuvieron a partir del programa informático ATLAS TI, con la finalidad de ordenar los datos conseguidos en las entrevistas aplicadas y las planificaciones analizadas.

En primera instancia, se llevó a cabo un detallado análisis, línea a línea, necesario al comienzo de un estudio para generar categorías iniciales (con sus propiedades y dimensiones) y para sugerir las relaciones entre ellas. Posteriormente, los datos se reagruparon entre las categorías de contenidos, estrategias y enfoque de enseñanza; materiales concretos utilizados y habilidades que se potencian; y subcategorías, como base para elaborar explicaciones más precisas y completas del fenómeno, concretizadas con la creación de las Networks, empleando el programa antes señalado.

El análisis e interpretación de resultados se comienza a efectuar para responder a los dos primeros objetivos específicos propuestos para esta investigación: Identificar las metodologías de enseñanza y aprendizaje de la geometría, que utilizan docentes de segundo ciclo de la comuna de Temuco y Padre Las Casas y Describir las metodologías utilizadas por docentes para la enseñanza y el aprendizaje de la geometría, con la finalidad de determinar aquellos contenidos para los cuales se requiere proponer nuevas estrategias metodológicas.

De acuerdo a lo referido por los profesores, a sus planificaciones y fichas de trabajo (sistematizados en las Network), éstos en su totalidad aplicaron una estructura definida en sus clases de geometría, caracterizándose por dos momentos: el primero, con actividades en las cuales los maestros introdujeron los contenidos conceptuales, por medio de dictado, uso de recursos TICs, como las presentaciones en power point; copia de los contenidos desde los textos de estudio entregados por el Ministerio de Educación, y lectura de éstos en las fichas de trabajo. Samper et al (2003) definen tres tipos de tareas que deben realizar los estudiantes para favorecer un pensamiento geométrico, éstas son la conceptualización, investigación y demostración. La primera se refiere a la construcción de conceptos y de relaciones geométricas. Es importante aclarar que no se trata de definir objetos geométricos, sino de conceptualizarlos. Por ejemplo, si lo que se desea es que los alumnos construyan el concepto de cuadrilátero, no es suficiente, ni deseable, que en principio se dé la definición de cuadrilátero como polígono de cuatro lados y se ilustre dibujando varios cuadriláteros, creyendo que con ello el alumno aprenderá lo que son estas figuras. Es preciso referir que los docentes ocuparon en sus clases actividades de definición de conceptos y dibujo de ellos, comprobando que en sus prácticas pedagógicas no consideraron llevar a cabo actividades de conceptualización.

En base al análisis anterior, se aprecia un tipo de enseñanza de la geometría ostensiva, esto es una cierta presentación de los objetos de enseñanza en la que todos los elementos y relaciones constitutivas de la noción prevista son proporcionados de un solo golpe por el profesor o el libro de texto. Claramente, el profesorado expuso en su discurso que al inicio de los contenidos se trabajan las definiciones, las que son requeridas para el posterior ejercicio procedimental de cada contenido geométrico. Es recomendable que los profesores incluyan representaciones gráficas (esquemas), lo que permitirá la apropiación de los contenidos conceptuales (Podestá, 2001). Vargas y Gamboa (2012) informan que en Geometría, el concepto está muy ligado a la imagen conceptual, que conviene enriquecer lo más posible. Por ejemplo, una actividad que permite una comprensión dinámica del concepto de altura, es formar en el geoplano un triángulo y, partiendo de él, cambiar sólo el vértice superior, encontrándose otros triángulos con la misma base y la misma medida de la altura. Con este 
Estudios Pedagógicos XLII, N 1: 87-105, 2016

ESTRATEGIAS METODOLÓGICAS PARA LA ENSEÑANZA Y EL APRENDIZAJE DE LA GEOMETRÍA, UTILIZADAS POR DOCENTES DE SEGUNDO CICLO, CON LA FINALIDAD DE GENERAR UNA PROPUESTA METODOLÓGICA ATINGENTE A LOS CONTENIDOS

tipo de actividad, se está trabajando el concepto de altura desde varias aristas, conociendo la variedad de éstas dependiendo del tipo de triángulo que se represente.

El modelo de razonamiento geométrico de Van Hiele abarca dos aspectos básicos: el descriptivo, mediante el cual se identifican diferentes formas de razonamiento geométrico de los individuos y se puede valorar su progreso; y el instructivo, el que marca pautas a seguir por los profesores para favorecer el avance de los estudiantes en el nivel de razonamiento geométrico en el que se encuentran (Vargas y Gamboa, 2012).

El aspecto instructivo presentado en este modelo, apunta a una primera fase de información, en la que se procede a tomar contacto con el nuevo tema u objeto de estudio. El profesor debe identificar los conocimientos previos y el nivel de razonamiento que puedan tener sus alumnos sobre este nuevo campo trabajo. Fouz y De Donosti (2005) citan a Ausubel (1978) para respaldar que este es el primer acercamiento a los conocimientos del alumno: "Si tuviera que reducir toda la Psicología Educativa a un sólo principio diría lo siguiente: el factor más importante que influye en el aprendizaje es lo que el alumno/a sabe. Averígüese esto y enséñese en consecuencia" (cit. en Fouz y De Donosti, 2005: 72). Los alumnos deben recibir información para conocer el campo de estudio que van a iniciar, los tipos de problemas que van a resolver, los métodos y materiales que utilizarán. Los docentes, al entregar la información conceptual, respondieron en parte a la primera fase instructiva del modelo, detectándose en la introducción de los nuevos contenidos, sin embargo, que no todos consideraron la activación de los conocimientos previos como una estrategia pertinente a usar en sus prácticas pedagógicas. Más bien, dos docentes refieren que ocuparon en sus clases la lluvia de ideas como estrategia para la activación de estos conocimientos.

El segundo momento que caracteriza la estructura de las sesiones de geometría, es la explicación y demostración de los contenidos procedimentales. Todos los profesores comunicaron el uso de la explicación de los contenidos a través de la demostración de los ejercicios de cada área de contenido, como una estrategia que aplicaron en las lecciones de geometría. Al realizar aquella estrategia, estos docentes están incorporando en sus prácticas una de las visiones del aprendizaje de las matemáticas en el aula: de la mente del maestro a la del aprendiz, mediante la explicación verbal y el ejercicio repetido. Con esta visión, el docente explica detenidamente, una y otra vez, lo que no se entienda y les entrega muchos ejercicios, hasta que vayan asimilando lo que se debe hacer. Es relevante mencionar que aquella visión no se vincula con el paradigma que declaran sustentar sus prácticas, el constructivismo. Para ello, deberían incorporar una visión del aprendizaje, de la formación de códigos simbólicos y operacionales, según la cual el aprendizaje se adquiere mediante la experiencia y la investigación personal, siendo una construcción individual en el seno de un grupo (Cofre, 1998).

Relacionando con el modelo de Van Hiele, específicamente con el aspecto instructivo de éste, se concluye que el profesorado, al referir que utilizó la explicación y la demostración como estrategia central de aprendizaje, han empleado la segunda fase que hace alusión a la orientación dirigida. En esta fase se guía a los estudiantes mediante actividades, con el fin de que éstos descubran y aprendan las diversas relaciones o componentes básicos de la red de conocimientos por formar. El profesor debe seleccionar cuidadosamente estos problemas y actividades y, cuando lo necesiten, orientar a sus alumnos hacia la solución. De acuerdo con Jaime (1993), esta fase es fundamental, ya que en ella se construyen los elementos básicos de la red de relaciones del nivel correspondiente. Al respecto, cita a Van 
Hiele (1986), quien señala que "[...] las actividades (de la segunda fase), si se seleccionan cuidadosamente, constituyen la base adecuada del pensamiento de nivel superior" (Jaime, 1993: 10). El papel del profesor resulta primordial en esta fase, ya que debe seleccionar las actividades adecuadas que permitan al estudiante aprender los conceptos, propiedades o definiciones fundamentales para el nuevo nivel de razonamiento. Respecto a la planificación de la fase dos, Vargas y Gamboa indican que "una planificación cuidadosa de la secuencia tendrá en cuenta la necesidad de conseguir pequeños éxitos que estimulen su autoestima y favorezcan una actitud positiva hacia las matemáticas" (Vargas y Gamboa, 2012: 36).

En base a lo expuesto por los docentes, sólo dos de ellos poseen un conocimiento del Modelo de Van Hiele, señalando los diferentes niveles. No obstante, declaran no conocer en qué nivel se encuentran sus estudiantes, pero que sí realizan la gran cantidad de actividades orientadas a los últimos niveles del pensamiento geométrico, caracterizándose por un nivel de razonamiento superior. Ello dificulta la apropiación y comprensión de los conocimientos de geometría de todos sus educandos, en especial de aquellos que se hallan en los primeros niveles, pues como indican estos autores, es preciso proponer actividades que respondan al nivel en el cual están los estudiantes, ya que sólo de esta forma será significativo el aprendizaje. Los profesores restantes expresan no conocer en qué consiste este modelo, por lo que sus actividades no están dirigidas a potenciar los niveles de visualización, análisis, ordenación o clasificación, deducción formal y rigor. Además, no poseen conocimientos respecto a cómo organizar las actividades de acuerdo a lo planteado por Van Hiele, es decir, iniciar con preguntas o información, orientación dirigida, explicación, orientación libre e integración. Al realizar un análisis de las clases de los profesores, se concluye que algunos de éstos emplean las preguntas e información, respondiendo a la activación de los conocimientos previos, siendo éste uno de los principios del aprendizaje significativo. Además, se aprecia la utilización de la fase 2, la cual señala que los estudiantes comienzan a explorar el campo de estudio resolviendo actividades y problemas propuestos por el docente; y la fase 3, actividades de explicación, es decir, que los estudiantes intercambien sus experiencias, comenten lo realizado y justifiquen sus puntos de vista. Sin embargo, carecen tanto de actividades de orientación libre, vale decir, que los educandos apliquen y combinen los conocimientos adquiridos en las fases anteriores para solucionar nuevas actividades, como de actividades de integración, es decir, actividades en las cuales adquieran una visión general de los contenidos y habilidades.

Al analizar si los profesores iniciaron la clase con actividades dinámicas, juegos o material concreto, uno de ellos aludió que al comenzar la lección, planteó preguntas de ingenio y la vinculación de los contenidos con la vida diaria. Los demás docentes, sólo indicaron que al empezar la clase activaron los conocimientos previos por medio de preguntas orientadas a los contenidos que trabajaron, lo que se puede demostrar de igual forma en las planificaciones anexadas, que dan cuenta de la estrategia empleada.

Para el desarrollo de las sesiones de trabajo, los docentes en su totalidad aplicaron guías de aprendizaje, textos de estudio entregado por el Ministerio de Educación y ejercicios que debieron copiar desde el pizarrón, siendo éstos los recursos centrales en sus clases de geometría. En estos tipos de recursos, predominaron los elementos icónicos y simbólicos; en cuanto a los materiales concretos, los docentes informan que ocuparon materiales como cuerpos y redes geométricas e instrumentos como la regla, compás, transportador y escuadra. Las prácticas de estos docentes se caracterizaron por una carencia del uso de materiales concretos que desarrollen los contenidos del área, siendo los 
Estudios Pedagógicos XLII, № 1: 87-105, 2016

ESTRATEGIAS METODOLÓGICAS PARA LA ENSEÑANZA Y EL APRENDIZAJE DE LA GEOMETRÍA, UTILIZADAS POR DOCENTES DE SEGUNDO CICLO, CON LA FINALIDAD DE GENERAR UNA PROPUESTA METODOLÓGICA ATINGENTE A LOS CONTENIDOS

recursos comunicados con anterioridad los que imperan en las clases de geometría. Como es anunciado por los docentes, la justificación para no ocupar otros materiales concretos es que no existen variedad para todos los contenidos, que no disponen en el establecimiento de ellos y si hay, éstos no alcanzan para todos los alumnos. Dos docentes, las que se realizaron clases en séptimo y octavo, dijeron que los estudiantes de estos niveles deben desarrollar la habilidad de abstracción, por lo que no necesitan los materiales concretos. Además, una de ellas informa que los educandos se encuentran en un nivel de pensamiento geométrico de clasificación, que implica trabajar actividades sin el soporte concreto, a pesar de que se sabe que en las aulas hay diversidad de estudiantes y características variadas.

Las actividades que se apreciaron en las guías de aprendizaje y textos de estudio, se identificaron por desarrollar la habilidad de visualización. Este tipo de actividades es de razonamiento, y se basa en el uso de elementos visuales o espaciales, tanto mentales como físicos, utilizados para resolver problemas o probar propiedades (García y López, 2008). La utilización de recursos pictóricos para desarrollar los contenidos de geometría fue un recurso pertinente en sus prácticas, ya que esta disciplina es eminentemente visual, pues cada concepto geométrico es reconocido y comprendido a través de la visualización. Cabe aclarar que, si bien la habilidad de visualización es un primer acercamiento a los objetos geométricos, no se puede aprender la geometría sólo viendo una figura u otro objeto geométrico. La generalización de las propiedades o la clasificación de las figuras no pueden darse a partir únicamente de la percepción. Es necesario que el alumno se enfrente a diversas situaciones donde los conocimientos adquieran sentido, por ejemplo, a través de las construcciones geométricas, en las que se puede variar el tipo de información que se les entrega. En base a lo anterior, tanto en su discurso como en sus fichas de trabajo, se apreció en el profesorado el uso de actividades de construcción, con la finalidad de adquirir los conceptos enseñados y los procedimientos a aprender. Este tipo de habilidad de dibujo, está relacionada con las reproducciones o construcciones gráficas que los alumnos hacen de los objetos geométricos. La reproducción se refiere a la copia de un modelo dado, ya sea del mismo tamaño o a escala, cuya construcción puede realizarse en base a la información que se da en forma verbal (oral o escrita) o gráfica. Las actividades de construcción son de gran riqueza didáctica, debido a que promueven en el alumno su capacidad de análisis de los elementos dibujados, al buscar las relaciones y propiedades que están dentro de su construcción. La construcción de figuras no sólo es un propósito de la enseñanza de la geometría sino que, además, constituye un medio para que los alumnos sigan explorando y profundizando en los conocimientos que ya tienen e incluso construyan otros nuevos (García y López, 2008). Los docentes, al ocupar en sus prácticas los instrumentos geométricos, promovieron su uso, constituyendo una herramienta indispensable en la enseñanza de la geometría, porque permiten desarrollar en los estudiantes sus destrezas para utilizarlos y sus habilidades de dibujo. Al pedir a los alumnos que, usando sus instrumentos geométricos, reproduzcan una figura, éstos tuvieron que identificar las figuras involucradas y la manera en que están relacionadas dentro de la configuración completa, con lo cual estuvieron desarrollando su habilidad de visualización.

En base a lo analizado, una docente informó que desarrolló actividades de demostración, lo que se ve respaldado en sus guías de aprendizaje. Los estudiantes debieron elaborar conjeturas sobre los procedimientos de resolución de problemas de transformaciones isométricas, en la cuales tuvieron que explicar, a partir de argumentos, cómo resolvieron las situaciones problemáticas. Con este tipo de actividades, los educandos socializan el 

ATINGENTE A LOS CONTENIDOS

conocimiento geométrico, ya que desde el enfoque de resolución de problemas se concibe al conocimiento como una construcción social. Las tareas de demostración son esenciales en geometría y deben estar presentes en la interacción del aula escolar; la construcción de argumentos lógicos es una habilidad que forma parte esencial de la cultura geométrica y es deseable que todos los alumnos la desarrollen. A partir de estas actividades de demostración, esta docente desarrolló la habilidad de comunicación, la que se refiere a que el alumno sea capaz de interpretar, entender y comunicar información geométrica, ya sea en forma oral, escrita o gráfica, usando símbolos y vocabulario propios de la geometría. Las habilidades del lenguaje están estrechamente relacionadas con el pensamiento y están presentes en muchos sentidos durante las clases de Matemáticas y de geometría en particular, por ejemplo, cuando se lee e interpreta la información de un problema para empezar a resolverlo; se discute con los compañeros de equipo las posibles estrategias de resolución; se presenta ante el grupo el resultado y procedimiento que se siguió para resolver un problema; se justifica un resultado o un procedimiento; se valida una conjetura que se hizo (García y López, 2008).

Algunos docentes incorporaron la resolución de problemas en sus clases de geometría, estrategia aplicada en el momento final de la clase. Al analizar el tipo de problemas que presentan los docentes, éstos son no rutinarios, caracterizado por un alto nivel de abstracción, lo que sin duda, no es pertinente para los educandos que se encuentren en los niveles de visualización o análisis, sino más bien para los niveles de clasificación, deducción formal y rigor. Para que aquella estrategia sea pertinente, en primera instancia se debiese conocer cuál es el nivel del pensamiento geométrico en el cual se encuentra cada estudiante para, de esta forma, presentar problemas que sean atingentes a sus características cognitivas. Al emplear los problemas geométricos, se favoreció la habilidad de aplicación y transferencia; los estudiantes fueron capaces de aplicar lo aprendido en las sesiones de trabajo, al resolver los problemas propuestos por los docentes. No obstante, esta habilidad no sólo se refiere a aplicar lo aprendido a los problemas planteados dentro de la misma geometría, sino también a modelar geométricamente situaciones del mundo físico o de otras disciplinas. La transferencia puede darse de varias maneras: puede ser que el alumno transfiera el contenido aprendido en geometría para resolver otra tarea que también pertenece al ámbito matemático, como el álgebra; o bien, que transfiera lo aprendido en geometría a una tarea que pertenece a otra área del conocimiento, como la física, en cuyo caso se habla de la aplicación de las Matemáticas.

A continuación se presenta la Network creada a partir de la entrevista realizada al docente $\mathrm{N}^{\circ} 1, \mathrm{~N}^{\circ} 2, \mathrm{~N}^{\circ} 3$ y $\mathrm{N}^{\circ} 4$, evidenciando contenidos, estrategias y enfoque de enseñanza, materiales concretos utilizados y habilidades que se potencian. 
Estudios Pedagógicos XLII, $\mathrm{N}^{\circ}$ 1: 87-105, 2016

ESTRATEGIAS METODOLÓGICAS PARA LA ENSEÑANZA Y EL APRENDIZAJE DE LA GEOMETRÍA, UTILIZADAS POR DOCENTES DE SEGUNDO CICLO, CON LA FINALIDAD DE GENERAR UNA PROPUESTA METODOLÓGICA ATINGENTE A LOS CONTENIDOS

Fig. $N^{\circ}$ 1. Contenidos, estrategias y enfoques de enseñanza, materiales concretos empleados y habilidades que potencian el docente de quinto básico.

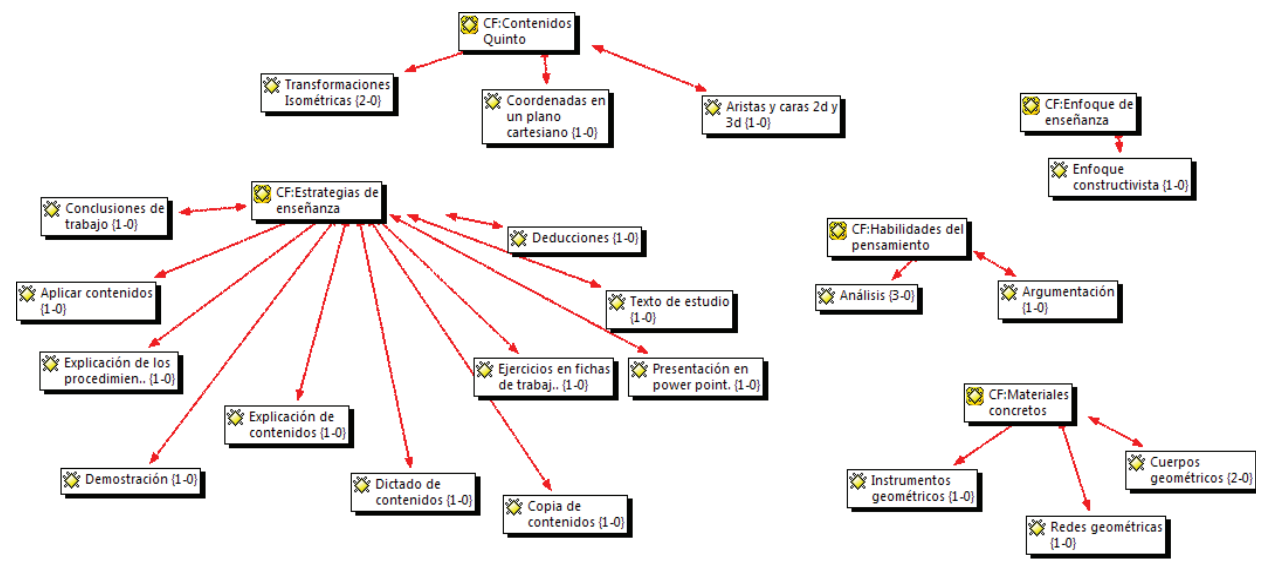

Como se aprecia en la Figura n ${ }^{\circ}$, el docente de quinto básico desarrolló los contenidos de transformaciones isométricas, coordenadas en un plano cartesiano y el análisis de aristas y caras en figuras $2 \mathrm{~d}$ y $3 \mathrm{~d}$. Esta figura respalda las estrategias de enseñanza-aprendizaje que ocupó el docente, las cuales apuntan al trabajo de los contenidos conceptuales, por medio del dictado de éste y la copia de aquellos contenidos por los estudiantes. En un segundo momento, efectuó la explicación y demostración de los procedimientos de cada área de contenido y la aplicación de aquellos en actividades propuestas en fichas de trabajo y textos de estudio, extrayendo conclusiones y deducciones. Incorporó los materiales concretos de cuerpos y redes geométricas e instrumentos geométricos: regla, compás, transportador y escuadra. Cabe informar, que el profesor alude a, y profesa, un paradigma constructivo en sus clases de geometría.

Fig. $N^{\circ}$ 2. Contenidos, estrategias y enfoques de enseñanza, materiales concretos ocupados y habilidades que potencia el docente de sexto básico

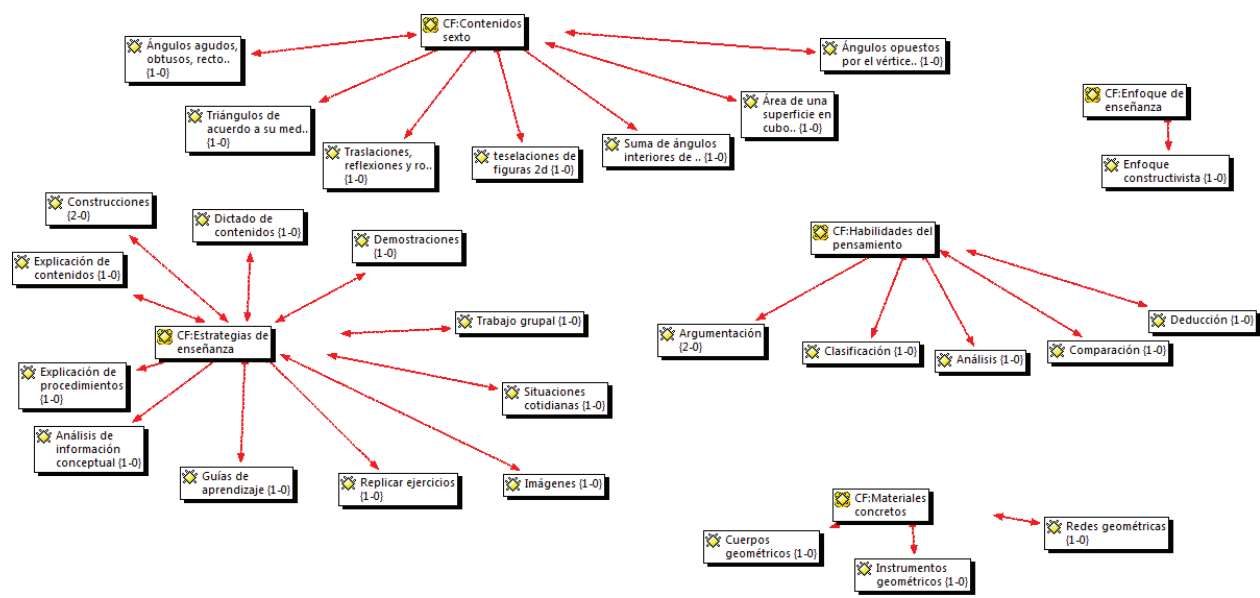



ATINGENTE A LOS CONTENIDOS

La Figura $n^{\circ} 2$ muestra los contenidos que trabajó el profesor de sexto básico en sus sesiones de geometría, siendo éstos: tipos de ángulos y triángulos, trasformaciones isométricas, teselaciones, ángulos interiores de un triángulo y áreas de cuerpos poliedros. Las estrategias de enseñanza-aprendizaje que comunicó son aquellas orientadas a los contenidos conceptuales, como dictados, explicación y análisis de éstos. Los procedimientos fueron trabajados a partir de las demostraciones, explicaciones, construcciones y réplicas de los ejercicios, ocupando actividades de situaciones cotidianas e imágenes, plasmadas en guías de aprendizaje. Las tareas de aprendizaje pretendieron desarrollar las habilidades de argumentación, clasificación, análisis y comparación, bajo un paradigma constructivista. El apoyo concreto usado fueron los cuerpos y redes geométricas, e instrumentos geométricos.

Fig. $N^{\circ}$ 3. Análisis de planificación docente sexto básico.
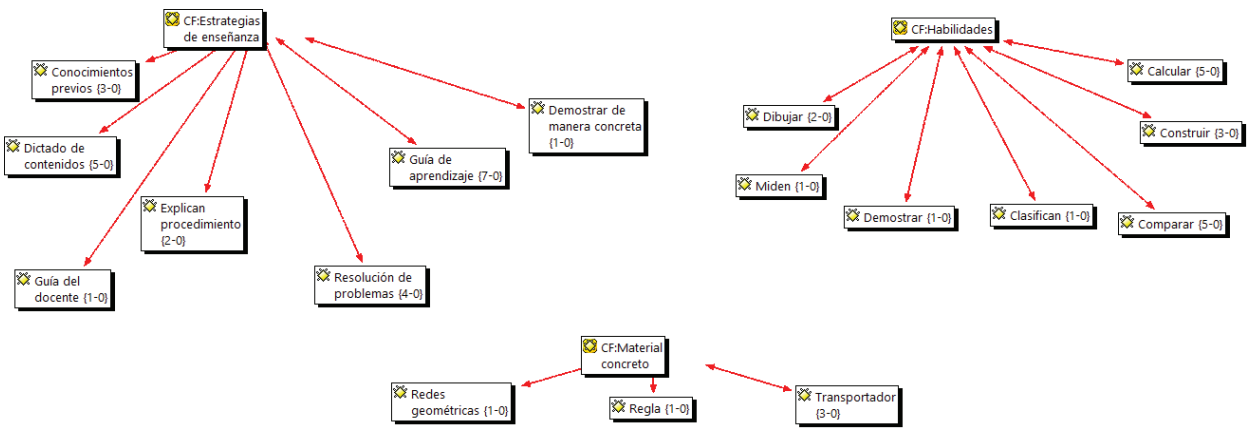

En la Figura $\mathrm{N}^{\circ} 3$ se logran observar las estrategias de enseñanza que lleva a cabo el docente de sexto básico, las cuales apuntan a la activación de los conocimientos previos, dictado de contenidos, explicación de procedimientos y guía del docente en los ejercicios propuestos, demostración de manera concreta, resolución de problemas y la aplicación de guías de aprendizaje. Cabe mencionar el uso de material concreto, como las redes geométricas, e instrumentos, como la regla y el transportador. Las habilidades que trabajó fueron el dibujo, la medición, la demostración, la clasificación, la comparación, la construcción y el cálculo. 
Estudios Pedagógicos XLII, N 1: 87-105, 2016

ESTRATEGIAS METODOLÓGICAS PARA LA ENSEÑANZA Y EL APRENDIZAJE DE LA GEOMETRÍA, UTILIZADAS POR DOCENTES DE SEGUNDO CICLO, CON LA FINALIDAD DE GENERAR UNA PROPUESTA METODOLÓGICA ATINGENTE A LOS CONTENIDOS

Fig. $N^{\circ}$ 4. Contenidos, estrategias y enfoques de enseñanza, materiales concretos utilizados y habilidades que potencian la docente de séptimo y octavo básico
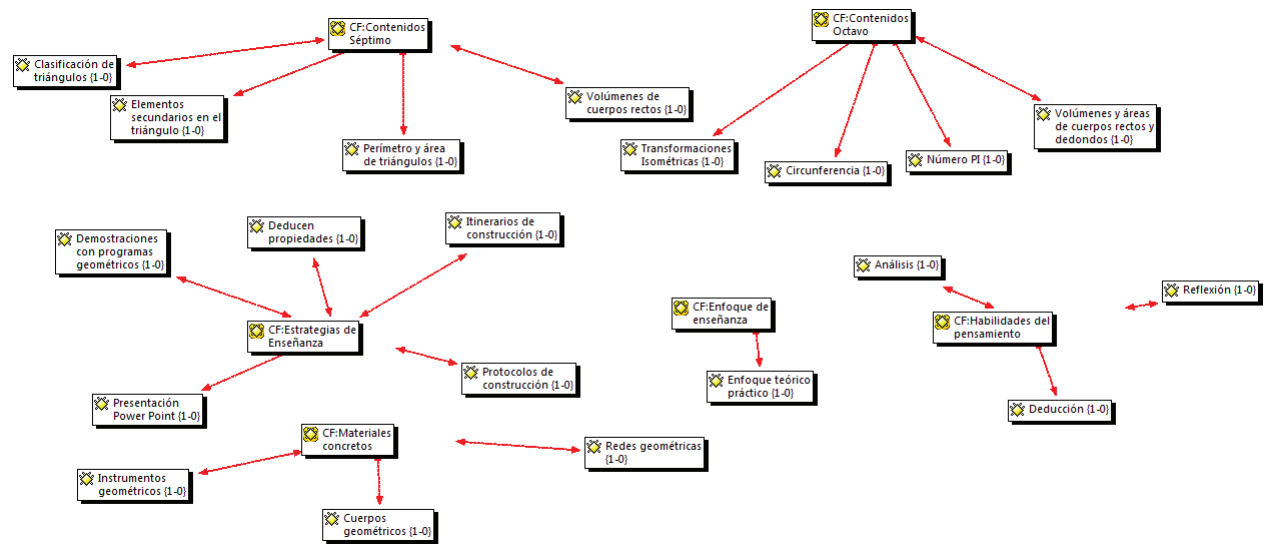

La Figura $\mathrm{N}^{\circ} 4$ muestra los contenidos que trabajó la profesora en los cursos de séptimo y octavo básico. En el primero, se desarrollan los contenidos de clasificación, elementos secundarios, perímetro y áreas de triángulos y volúmenes de cuerpos rectos. En octavo básico, se ven transformaciones isométricas, circunferencias, número pi y volúmenes y áreas de cuerpos redondos. Las estrategias de enseñanza y aprendizaje implementadas fueron presentaciones power point, protocolos e itinerarios de construcción, deducción de propiedades y demostraciones. Se desarrollaron las habilidades de análisis, deducción y reflexión, bajo un enfoque teórico-práctico. Los materiales utilizados fueron cuerpos y redes geométricas e instrumentos. 


\section{PROPUESTA METODOLÓGICA} ATINGENTE A LOS CONTENIDOS

Por medio de los resultados analizados, se concluye que los docentes orientaron sus prácticas hacia la enseñanza tradicional, en la cual priman actividades en fichas de trabajo, así como una visión de aprendizaje donde el docente explica los contenidos, entrega ejemplos y los educandos reproducen lo observado, proponiéndose diferentes actividades que respondan a actividades lúdicas, utilizando material concreto, considerando el modelo propuesto por Van Hiele (se presentan dos ejemplos de actividades).

A continuación se presentan actividades para trabajar Teselaciones con materiales 3D.

\begin{tabular}{|l|c|}
\hline \multicolumn{3}{|c|}{ Transformaciones Isométricas } \\
\hline \multicolumn{3}{|c|}{ Contenidos: Teselaciones } & $\begin{array}{c}\text { Materiales: cubos, materiales reciclables, } \\
\text { imagen de panal de abeja. }\end{array}$ \\
\hline \multicolumn{3}{|c|}{ ACTIVIDADES } \\
similitudes y diferencias de trabajar con objetos bidimensionales. \\
$\checkmark \quad$ Comienza con los cubos. ¿Puedes apilar los cubos para llenar el \\
espacio tridimensional sin dejar espacios? Para probar esto, observa \\
si hay algún espacio entre los cubos lo suficientemente grande para \\
meter un lápiz. Si no hay ninguno, creaste un espacio tridimensional \\
teselado. \\
$\checkmark \quad$ Ahora prueba con cada una de las otras formas. ¿Con \\
cuáles se crea un espacio teselado? ¿Con cuáles no? \\
$\checkmark \quad$ ¿Con qué otras formas, de la naturaleza o de la vida diaria, se te \\
ocurre que se pueden crear espacios teselados? ¿Se te ocurren formas \\
que no crearían espacios teselados? \\
$\checkmark \quad$ ¿Qué ocurre? \\
¿Por qué crees que ciertos objetos—como cajas de cereal o celdas de \\
panal crean espacios teselados? Al crear un espacio teselado, lo llenas \\
de la manera más eficiente. Por ejemplo, si puedes usar teselado para \\
empacar cajas en un camión o en un anaquel de supermercado, podrás \\
empacar tantas cajas como sea posible. De forma similar, las abejas \\
utilizan todo el espacio disponible en su colmena construyendo su \\
panal en un patrón teselado.
\end{tabular}


Estudios Pedagógicos XLII, N 1: 87-105, 2016

ESTRATEGIAS METODOLÓGICAS PARA LA ENSEÑANZA Y EL APRENDIZAJE DE LA GEOMETRÍA, UTILIZADAS POR DOCENTES DE SEGUNDO CICLO, CON LA FINALIDAD DE GENERAR UNA PROPUESTA METODOLÓGICA ATINGENTE A LOS CONTENIDOS

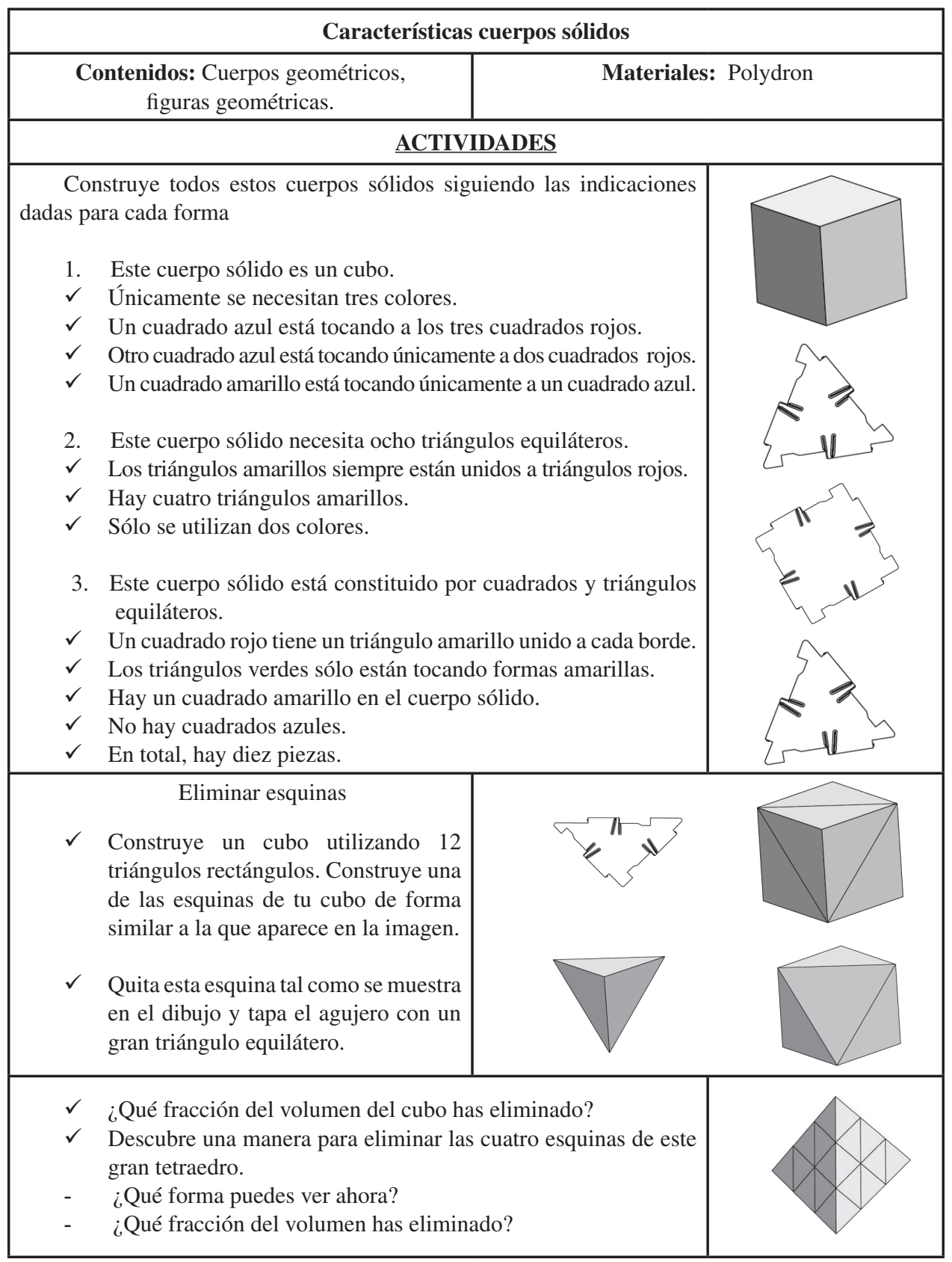




\section{CONCLUSIONES} ATINGENTE A LOS CONTENIDOS

A partir del análisis realizado en torno a las estrategias que ocuparon los docentes de segundo ciclo en la asignatura de Matemática, y específicamente el eje temático de Geometría, la propuesta metodológica levantada y los objetivos formulados, se destacan las siguientes conclusiones:

La estructura de la clase de geometría se caracterizó por dos momentos: el primero, de introducción de contenidos conceptuales, por medio de la definición de los conceptos y dibujo de ellos. No obstante, se observa que éstas carecen de elementos gráficos, como esquemas que los sistematicen, y no responden a las tareas de conceptualización.

Algunos docentes declararon no conocer el modelo propuesto por Van Hiele, en cuanto a los niveles del pensamiento geométrico (visualización, análisis, clasificación, deducción forma y rigor) y las fases de enseñanza (preguntas, orientación dirigida, explicación orientación libre e integración).

Hay docentes que informaron conocer los niveles del pensamiento geométrico, sin embargo, expresaron no conocer en qué nivel se encuentran todos sus estudiantes, por lo cual sus actividades no desarrollan de forma explícita un nivel u otro.

Algunos docentes expresan que sus actividades responden a los últimos niveles del pensamiento geométrico, es decir, deducción formal y rigor, realizando actividades de resolución de problemas, los cuales carecen de elementos gráficos y concretos.

Los profesores ocuparon en sus clases de geometría la fase 1, de preguntas e información, y la fase 2, de orientación dirigida; en menor porcentaje, la fase 3 de explicación. Señalan no utilizar la fase de orientación libre e integración.

Los docentes, al utilizar la fase 1 del modelo de Van Hiele, activan los conocimientos previos de los educandos, cumpliendo con el paradigma que señalan utilizar en sus prácticas pedagógicas, siendo éste el constructivismo.

La fase de enseñanza que primó en sus clases de geometría es la orientación dirigida, es decir, ellos enseñan el contenido y luego éste es replicado por los estudiantes de forma similar al docente, cumpliendo con una de las visiones del aprendizaje en el aula, de la mente del maestro a la mente del aprendiz, lo cual no responde al paradigma constructivista que declaran utilizar.

La resolución de problemas es utilizada por algunos docentes como estrategia para transferir los conocimientos, los cuales no responden a las características del pensamiento de los educandos, pues son seleccionados para que puedan ser resueltos por estudiantes que se encuentren en los últimos niveles del pensamiento geométrico.

Las actividades que presentaron un mayor porcentaje de uso, son aquellas en las cuales se utilizan fichas de trabajo y el texto de estudio entregado por el Ministerio de Educación.

El profesorado usó pocos materiales concretos para trabajar los contenidos geométricos, siendo sólo los cuerpos y redes geométricas, como también los instrumentos geométricos: compás, transportador, escuadra y regla.

El aprendizaje de la geometría debe ir de lo concreto a lo abstracto, por lo cual es pertinente que se desarrollen actividades con materiales concretos como geoplanos, tangramas, pentominos, cubos somas, libros de espejos, bloques poligonales, entre otros.

La propuesta metodológica planteada se basó en actividades que incorporan materiales concretos (antes señalados), así como actividades lúdicas y de motivación, para responder a los distintos niveles del pensamiento geométrico en el cual se encuentran los educandos. 
Estudios Pedagógicos XLII, N 1: 87-105, 2016

ESTRATEGIAS METODOLÓGICAS PARA LA ENSEÑANZA Y EL APRENDIZAJE DE LA GEOMETRÍA, UTILIZADAS POR DOCENTES DE SEGUNDO CICLO, CON LA FINALIDAD DE GENERAR UNA PROPUESTA METODOLÓGICA ATINGENTE A LOS CONTENIDOS

Las actividades propuestas desarrollan las habilidades visuales, en las cuales existe coordinación visomotora, constancia perceptual y memoria visual; habilidades de comunicación, recolección e interpretación de información, denominación, definición, escucha, registro y lectura; habilidades lógicas o de razonamiento, donde deben clasificar, comparar y justificar; y de aplicación o transferencia.

La propuesta metodológica responde a las cinco fases del aprendizaje del modelo de Van Hiele: preguntas/información, orientación dirigida, explicación, orientación libre e integración.

En cuanto a las limitaciones de este estudio, se debe afirmar que éstas radican en que es una investigación llevada a cabo con una unidad de análisis intencionada, reducida, por lo que los hallazgos, si bien dan cuenta de una realidad, no se pueden generalizar.

Al no ser factible realizar la observación de las clases de geometría, debido a que aquel contenido no fue abordado en los tiempos de esta investigación, los resultados sólo fueron triangulados a partir de las entrevistas, planificaciones y guías de aprendizaje, lo que limitó los hallazgos del estudio.

Otra carencia de este trabajo fue el no incluir a los estudiantes como participantes de la investigación, lo que hubiese permitido tener la mirada de éstos, referente a las estrategias desarrolladas por los docentes, evaluando de igual forma lo atractivo y motivante que resultaron las actividades llevadas a cabo.

En lo referente a las proyecciones de este estudio, se pueden indicar que cualquier futura investigación debiese considerar dentro de sus objetivos conocer la motivación que presentan los estudiantes en las clases desarrolladas por los docentes, pues de esta forma se evaluaría el impacto que pueden tener las clases innovadoras en la motivación que muestren los alumnos por el aprendizaje matemático.

Con esta investigación se podría motivar a los docentes a interiorizarse en el modelo expuesto por Van Hiele, trabajando en base a los postulados descriptivos e instructivos, permitiendo desarrollar un pensamiento geométrico en todos sus educandos.

Se propone, por tanto, ampliar la investigación a través de la implementación de la propuesta metodológica, primeramente evaluando a los estudiantes para identificar en qué nivel del pensamiento geométrico se encuentran para, de esta forma, llevar a cabo estrategias y actividades que respondan a sus características cognitivas. Esto supone también incluir la observación directa a las sesiones de geometría, lo que permitiría tener argumentos más sólidos sobre los resultados obtenidos, y triangular con los instrumentos antes señalados.

\section{REFERENCIAS BIBLIOGRÁFICAS}

Apostá, P. (2011). Geometría. Consulado 14 de noviembre de 2013 de http://www.uv.es/ ivorra/ Libros/Geometria.pdf

Bressan, A. Bogisic, B. y Crego, K. (2010). Razones para enseñar geometría en la educación básica. Mirar, construir, decir y pensar. Consultado 15 de noviembre de 2013 desde http://www. noveduc.com/index.php

Cofré, A. y Tapia, L. (1998). Cómo desarrollar el razonamiento matemático. Santiago: Editorial Universitaria.

Duval, R. (2001). La Geometría desde un Punto de Vista Cognitivo. Consultado 11 de noviembre de 2013 desde http://fractus.uson.mx/Papers/ICMI/LaGeometria.htm

Foz, F. y Donoso, B. (2005). Modelo de Van Hiele para la didáctica de la Geometría. Consultado 
Estudios Pedagógicos XLII, N 1: 87-105, 2016 ESTRATEGIAS METODOLÓGICAS PARA LA ENSEÑANZA Y EL APRENDIZAJE DE LA GEOMETRÍA, UTILIZADAS POR DOCENTES DE SEGUNDO CICLO, CON LA FINALIDAD DE GENERAR UNA PROPUESTA METODOLÓGICA ATINGENTE A LOS CONTENIDOS

10 de mayo de 2014 desde http://www.cimm.ucr.ac.cr/ciaem/articulos/universitario/ materiales/Modelo\%20de\%20Van\%20Hiele\%20para\%201a\%20did\%C3\%A1ctica\%20 de\%201a\%20Geometr\%C3\%ADa.*Fouz,\%20Fernando\%3B\%20\%20De\%20Donosti,\%20 Berritzegune.*Fernando\%20Fouz,\%20Berritzegune\%20de\%20Donosti.pdf

Jaime, A. (1993). Aportaciones a la interpretación y aplicación del modelo de Van Hiele: La enseñanza de la isometría en el plano. La evaluación del nivel de razonamiento. Consultado 15 de junio de 2014 desde http://www.uv.es/gutierre/archivos1/textospdf/Jai93.pdf

Latorre, A (2003). La investigación Acción: Conocer y cambiar la práctica educativa. Barcelona: Grao.

López, O. García, S. (2008). La Enseñanza de la Geometría. Consultado 6 de noviembre de 2013 desde http://www.inee.edu.mx/mape/themes/TemaInee/Documentos/mapes/geometriacompletoa.pdf

Rivero, M. y Zanocco, P. (1988). Matemática: un juego de niños. Santiago: Universidad Católica de Chile

Sandín, E. (2003). Investigación cualitativa en educación. Madrid: MacGraw-Hill

Vargas, G. y Gamboa, R. (2012). El modelo de Van Hiele y la enseñanza de la geometría. Uniciencia, 27 (1), 74-94.

Villella, J. (2001). Uno, dos, tres... Geometría Otra Vez. Buenos Aires: Aique 
